\title{
Slow and fast dynamics in coupled systems: A time series analysis view
}

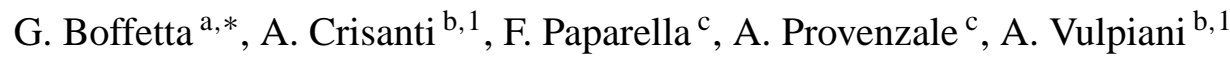 \\ a Dipartimento di Fisica Generale, Università di Torino, via Pietro Giuria 1, 10125 Torino, Italy \\ b Dipartimento di Fisica, Università di Roma "La Sapienza", p.le Aldo Moro 2, 00185 Roma, Italy \\ ${ }^{\mathrm{c}}$ Istituto di Cosmogeofisica del CNR, corso Fiume 4, 10133 Torino, Italy
}

Received 18 April 1997; received in revised form 29 September 1997; accepted 7 November 1997

Communicated by C.K.R.T. Jones

\begin{abstract}
We study the dynamics of systems with different timescales, when access only to the slow variables is allowed. We use the concept of finite size Lyapunov exponent (FSLE) and consider both the cases when the equations of motion for the slow components are known, and the situation when a scalar time series of one of the slow variables has been measured. A discussion on the effects of parametrizing the fast dynamics is given. We show that, although the computation of the largest Lyapunov exponent can be practically infeasible in complex dynamical systems, the computation of the FSLE allows to extract information on the characteristic time and on the predictability of the large-scale, slow-time dynamics even with moderate statistics and unresolved small scales. Copyright (C) 1998 Elsevier Science B.V.
\end{abstract}

\section{Introduction}

In the last two decades, the problem of extracting information from a measured time series has been studied extensively, see e.g. [1-12]. Several attempts have been devoted to the issue of distinguishing between deterministic and stochastic behavior, where "deterministic" has to be interpreted as "dominated by a small number of excited modes" and "stochastic" as "dominated by a large number of excited degrees of freedom". Once the presence of low-dimensional chaotic dynamics is assessed, various methods have been devised for determining the statistical properties of the attractor and to build appropriate models for either predicting or describing the system evolution.

Most methods for determining dynamical properties from measured signals are based on a procedure of phasespace reconstruction. Following the work of Packard et al. [6] and Takens [7], the so-called time-embedding techniques have been developed to address this problem. Their use (e.g. via delay coordinates) allows, at least in

\footnotetext{
* Corresponding author. Also at: Istituto Nazionale di Fisica della Materia, Unità di Torino.

${ }^{1}$ Also at: Istituto Nazionale di Fisica della Materia, Unità di Torino.
} 
principle, the determination of the dimensions [8], the Kolmogorov-Sinai entropy [9] and the Lyapunov exponents $[5,10]$ of the system by the analysis of a time series of just one scalar variable.

Unfortunately, this approach may have severe limitations in many practical situations. For example, the length of the time series is a crucial point in order to obtain reliable estimates of the phase-space properties of the system $[11,12]$. Further, there are simple stochastic processes that mimic a "false positive" answer to the search for lowdimensional chaotic dynamics, providing a finite value of the dimension under time-embedding in most practical cases [13-15]. Analogously, simple systems characterized by on/off intermittency require additional care in the procedure of phase-space reconstruction and analysis [16,17].

Another problem is encountered in systems with many different timescales. In this case, it has been shown [18,19] that the Lyapunov exponents may have a rather marginal role. The growth of a non-infinitesimal perturbation is indeed ruled by a nonlinear mechanism which depends on the details of the system. For this reason, despite the positiveness of the largest Lyapunov exponent, it is possible to have a long predictability time for some specific degrees of freedom. A typical example of this type of behavior is provided by three-dimensional turbulence, that is characterized by the contemporary presence of a hierarchy of eddy turnover times. In this case, large-scale motions have a predictability time that is much larger than the one suggested by the value of the largest Lyapunov exponent. In such a situation, the predictability time for realistic perturbations may thus have no relationship with the growth rate of infinitesimal perturbations.

As an attempt to overcome this problem, the concept of maximum Lyapunov exponent has recently been generalized in [19] to the case of non-infinitesimal perturbations, introducing the notion of the finite size Lyapunov exponent (FSLE). In this work, we further elaborate on this issue and apply this method to the detection of "large scale" ("slow") dynamical properties of measured systems characterized by the contemporary presence of different timescales. In particular, we are concerned with systems that can be separated into a slow part, $S$, described by the phase-space variables $\mathbf{x}_{\mathrm{s}}$, and a fast part $F$, described by the variables $\mathbf{x}_{\mathrm{f}}$. The two subsystems are coupled through a term of typical strength $\epsilon$. In the limit $\epsilon \rightarrow 0$, each of the two subsystems evolves independently with its own (chaotic) dynamics. The Lyapunov exponents of the slow and fast subsystem are $\lambda_{\mathrm{s}}<\lambda_{\mathrm{f}}$, respectively.

As for the coupling, we can either have a situation where the fast subsystem drives the slow one without being influenced by the latter, see e.g. [16,17], or a more generic coupling between the two parts [18,20]. For the specific application we are concerned with here, the form of the coupling is not very important. Preliminary results on the predictability of a slow system $S$ coupled with a faster system $F$ have been discussed in the case of two coupled Lorenz models [20]. In that work, the dynamics of the fast system was supposed to be known with arbitrary accuracy, and it was found that even if the value of the Lyapunov exponent is determined by the fast dynamics, the predictability of the slow system is dominated by its own characteristic time and it is almost unaffected by a small coupling with the fast dynamics.

Physically, we may think of the fast subsystem as representing small scales that, both in real experiments and numerical simulations, are not resolved. Consistent with this interpretation, here we assume that the dynamics of the fast subsystem is poorly known, and investigate the effects of parametrizing the fast dynamics when one has access only to the slow dynamics. In this framework, we consider two different situations. In the first case, the equations of motion of the slow system are given. In the second case, we consider the computation of the FSLE directly from the measured time series. The study of systems with two characteristic timescales is the first necessary step for the understanding of more realistic systems with several scales [21].

The remainder of this paper is organized as follows. In Section 2 we discuss the notion of FSLE introduced in [19]. In Section 3 we study the case of two coupled systems having different timescales, when access to the whole phase space of the slow system is allowed. In Section 4 we consider the same cases, but when just one scalar time series is supposed to have been measured. Section 5 gives conclusions and perspectives. 


\section{Extension of the Lyapunov exponent to finite perturbations}

Here we recall the basic ingredients and the algorithm for computing the FSLE, referring to [19] for further details. The definition of FSLE follows from that of error growing time $T_{r}(\delta)$ for a perturbation of size $\delta$. By definition, $T_{r}(\delta)$ is the time that a perturbation with initial size $\delta$ takes to grow by a factor $r$ during the system evolution. In general, the perturbation with size $\delta$ is supposed to be already aligned with the most unstable direction. The error ratio $r$ should not be taken too large, in order to avoid the growth through different scales. In many applications, $r=2$, so sometimes the $T_{r}$ is also called the error doubling time. The FSLE is defined from an ensemble average of predictability time according to

$$
\lambda(\delta)=\frac{1}{\left\langle T_{r}(\delta)\right\rangle} \ln r=\left\langle\frac{1}{T_{r}(\delta)}\right\rangle_{t} \ln r
$$

where $\langle\cdots\rangle_{t}$ denotes the natural measure along the trajectory and $\langle\cdots\rangle$ is the average over many realizations. The second equality comes from the definition of the time average along a trajectory for a generic quantity $A$ :

$$
\langle A\rangle_{t}=\frac{1}{T} \int_{0}^{T} A(t) \mathrm{d} t=\frac{\sum_{i} A_{i} \tau_{i}}{\sum_{i} \tau_{i}}=\frac{\langle A \tau\rangle}{\langle\tau\rangle} .
$$

in the particular case of $A=1 / \tau[19]$.

In the limit of infinitesimal perturbations, $\delta \rightarrow 0$, this definition reduces to that of the leading Lyapunov exponent $\lambda_{\max }$. In practice, $\lambda(\delta)$ displays a plateau at the value $\lambda_{\max }$ for sufficiently small $\delta$.

In most systems, the smaller scales evolve faster, as in the classic example of three-dimensional turbulent flows, and dominates the error growth for infinitesimal perturbations. When the size $\delta$ of the perturbation cannot be considered any longer infinitesimal, all the scales whose typical size is smaller than $\delta$ experience a diffusive separation and do not contribute to the exponential divergence in phase space. At this stage, the behavior of $\lambda(\delta)$ is governed by the nonlinear evolution of the perturbation, and, in general, $\lambda(\delta) \leq \lambda_{\max }$. The decrease of $\lambda(\delta)$ does follow a system-dependent law. In some cases, $\lambda(\delta)$ can be predicted by dimensional considerations. For the fully developed turbulence, e.g., dimensional considerations lead to the universal law $\lambda(\delta) \sim \delta^{-2}$ in the inertial range [19].

Therefore, the behavior of $\lambda$ as a function of $\delta$ contains important informations on the characteristic times governing the system, and it is a powerful tool for investigating the behavior of high-dimensional dynamical systems involving many characteristic scales in space and time.

To practically compute the FSLE, one has first to define a series of thresholds $\delta_{n}=r^{n} \delta_{0}$, and to measure the time $T_{r}\left(\delta_{n}\right)$ that a perturbation with size $\delta_{n}$ takes to grow up to $\delta_{n+1}$. The time $T_{r}\left(\delta_{n}\right)$ is obtained by following the evolution of the perturbation from its initial size $\delta_{\min }$ up to the largest threshold $\delta_{\max }$. This can be done, e.g., by integrating two trajectories of the system that start at an initial distance $\delta_{\min }$. In general, one must take $\delta_{\min } \ll \delta_{0}$, in order to allow the direction of the initial perturbation to align with the most unstable direction in the phase space. The FSLE, $\lambda\left(\delta_{n}\right)$, is then computed by averaging the predictability times over several realizations, see Eq. (1).

Note that the FSLE has conceptual similarities with the $\epsilon$-entropy [22]. This latter measures the bandwidth that is necessary for reproducing the trajectory of a system within a finite accuracy $\delta$. The $\epsilon$-entropy approach has already been applied to the analysis of simple systems and experimental data [23], giving interesting results. The direct calculation of the $\epsilon$-entropy, however, is much more expensive than that of the FSLE. This latter, in fact, is not more expensive than that of the largest Lyapunov exponent $\lambda_{\max }$. 


\section{FSLE and small-scale parametrization}

In this section we study the case of a slow system $S$, described by the variables $\mathbf{x}_{\mathrm{s}}$, coupled with a fast system $F$ described by the variables $\mathbf{x}_{\mathbf{f}}$. The equations of motion governing the slow variables are supposed to be known, and we study the effects of parametrizing the fast dynamics.

To study the evolution of the perturbation, we consider two trajectories $\mathbf{x}=\left(\mathbf{x}_{\mathrm{s}}, \mathbf{x}_{\mathrm{f}}\right)$ (reference) and $\mathbf{x}^{\prime}$ (perturbed) starting from two nearby locations in phase space. The perturbed trajectory is then made to evolve either according to the same equations as the reference one, or to modified equations, where the fast dynamics is replaced by a stochastic process, or simply neglected.

\subsection{Coupled maps}

The first example is provided by two coupled maps, namely

$$
\begin{array}{ll}
x_{\mathrm{s}}(n+1)=(1-\epsilon) f_{\mathrm{s}}\left[x_{\mathrm{s}}(n)\right]+\epsilon g\left[x_{\mathrm{s}}(n), x_{\mathrm{f}}(n)\right], & \bmod 1, \\
x_{\mathrm{f}}(n+1)=(1-\epsilon) f_{\mathrm{f}}\left[x_{\mathrm{f}}(n)\right]+\epsilon g\left[x_{\mathrm{f}}(n), x_{\mathrm{s}}(n)\right], & \bmod 1,
\end{array}
$$

where $f_{\mathrm{s}}$ and $f_{\mathrm{f}}$ are maps of the unit interval $[0,1]$ into itself. Here we use

$$
f_{\mathrm{s}}\left(x_{\mathrm{s}}\right)=\left.\mathrm{e}^{\lambda_{\mathrm{s}}} x_{\mathrm{s}}\right|_{\bmod 1}, \quad f_{\mathrm{f}}\left(x_{\mathrm{f}}\right)=\left.\mathrm{e}^{\lambda_{\mathrm{f}}} x_{\mathrm{f}}\right|_{\bmod 1}, \quad g\left(x_{\mathrm{s}}, x_{\mathrm{f}}\right)=\cos \left(2 \pi\left(x_{\mathrm{s}}+x_{\mathrm{f}}\right)\right),
$$

with $\lambda_{\mathrm{s}}<\lambda_{\mathrm{f}}$. Eqs. (3) completely define the dynamics of the system. We assume, however, that we can have access only to the slow variable $x_{\mathrm{s}}(n)$. The FSLE has thus to be computed only from the time evolution of $x_{\mathrm{s}}(n)$.

Before discussing how this can be achieved, we note that Eq. (1) is inadequate in the case of maps. Definition (1), in fact, tacitly assumes that we are able to determine the time when the size of the perturbation is exactly equal to the fixed threshold $\delta$. In the case of maps, this may not be possible. The appropriate definition in this case thus becomes (see [19])

$$
\lambda(\delta)=\frac{1}{\left\langle n_{r}\right\rangle}\left\langle\ln \left(\frac{\delta\left(n_{r}\right)}{\delta}\right)\right\rangle,
$$

where $\delta$ is the initial size of the perturbation and $\delta\left(n_{r}\right)$ is its size at the (discrete) time $n_{r}$. Here $n_{r}$ is the time at which the size of the perturbation first gets larger than (or equal to) $r \delta$, i.e., $\delta\left(n_{r}\right) \geq r \delta$ and $\delta\left(n_{r}-1\right)<r \delta$. The average $\langle\cdots\rangle$ is over an ensemble of many realizations, as in the original definition (1).

Let us now discuss how to compute $\lambda(\delta)$ from the knowledge of $x_{\mathrm{s}}(n)$. From a point $\left(x_{\mathrm{s}}, x_{\mathrm{f}}\right)$ on the system's attractor, we generate a new point representing the perturbed trajectory $\left(\delta_{\min } \ll 1\right)$

$$
x_{\mathrm{s}}^{\prime}=x_{\mathrm{s}}+\delta_{\min }, \quad x_{\mathrm{f}}^{\prime}=x_{\mathrm{f}}+\delta_{\min },
$$

and iterate the coupled maps for the original trajectory and the perturbed one. Note that, in this case, the perturbation has been applied to both the slow and the fast variables. We then compute $\lambda(\delta)$ from Eq. (5).

Fig. 1 shows the value of $\lambda(\delta)$ versus $\delta$ for the coupled maps system. The curve with filled triangles has been obtained by defining the distance in phase space as $\delta=\left|x_{\mathrm{s}}(n)-x_{\mathrm{s}}^{\prime}(n)\right|$. The curve denoted by the filled squares has been obtained with a different definition of distance, namely $\delta=\left\{\left[x_{\mathrm{s}}(n)-x_{\mathrm{s}}^{\prime}(n)\right]^{2}+\left[x_{\mathrm{s}}(n-1)-x_{\mathrm{s}}^{\prime}(n-1)\right]^{2}\right\}^{1 / 2}$ (reminiscent of the time-embedding procedure, see Section 3.2). Both curves are obtained by an average over $10^{4}$ samples for each value of $\delta$; analogous results are obtained with more limited statistics. For small $\delta$, the dynamics of the perturbation is driven by the fast mode, and $\lambda(\delta)$ tends toward $\lambda_{\max } \simeq \lambda_{\mathrm{f}}=0.5$. For large values of $\delta$, the growth of the perturbation is governed mainly by the slow dynamics and $\lambda(\delta)$ approaches $\lambda_{\mathrm{s}}=0.1$. The transition 


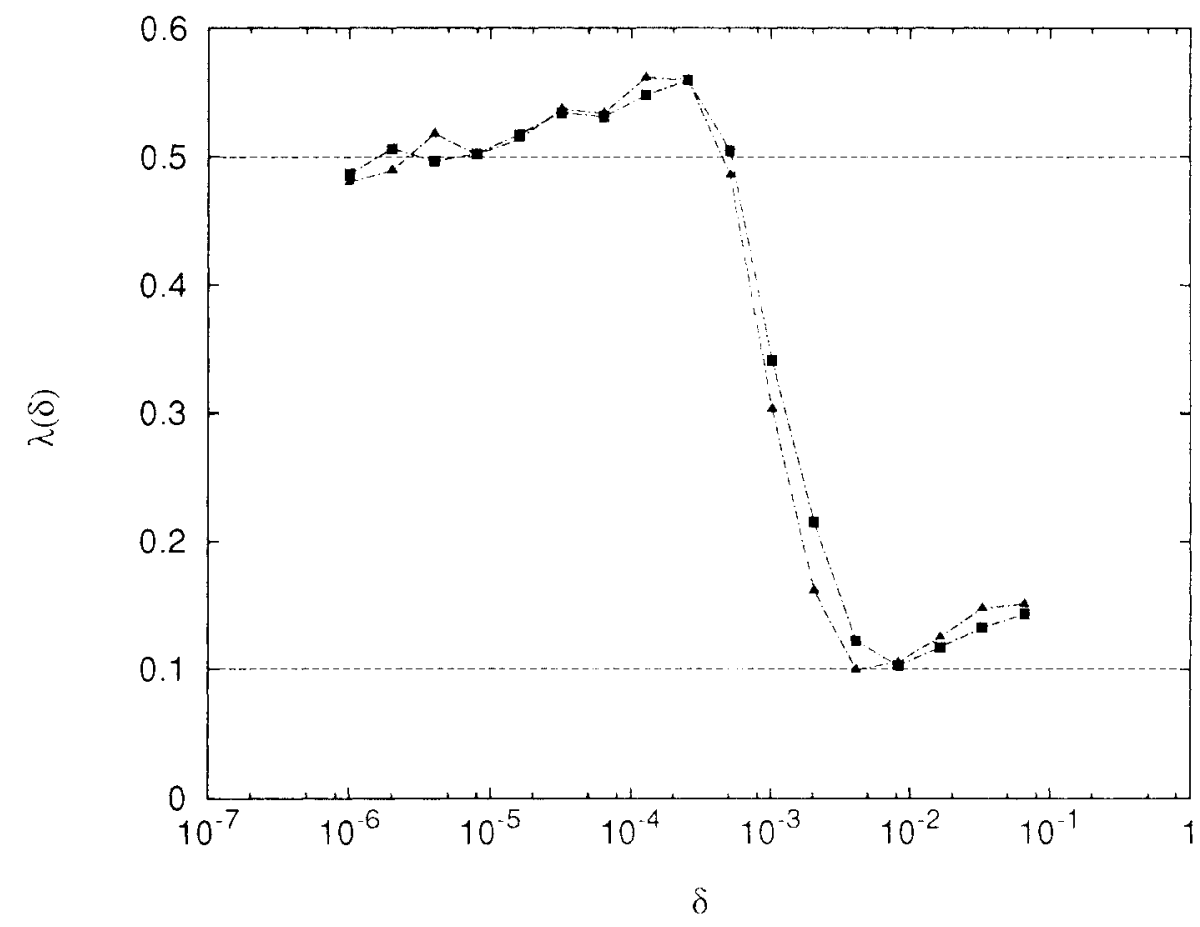

Fig. 1. The FSLE, $\lambda(\delta)$, as a function of $\delta$ for the coupled maps (3) and (4) with $\lambda_{\mathrm{s}}=0.1, \lambda_{\mathrm{f}}=0.5$ and $\epsilon=2 \times 10^{-3}$. The two curves refer to the different definitions of distance discussed in the text. The parameters of the perturbation are $\delta_{\min }=10^{-9}, \delta_{0}=10^{-6}, \delta_{\max }=0.1$, $r=2$ and the average is over $10^{4}$ realizations for each point in the FSLE curve. The horizontal lines indicate the values of $\lambda_{\mathrm{s}}$ and $\lambda_{\mathrm{f}}$.

between the two regimes takes place at $\delta \sim \epsilon=2 \times 10^{-3}$; changing the strength of the coupling modifies only the value of $\delta$ where the transition takes place. It is worth noting that the two definitions of distance used in obtaining Fig. 1 give almost coincident results. This indicates that, at least in this case, the definition of distance which is employed is not crucial, at variance with what happens in other cases (e.g. for on/off intermittent systems [17]).

As discussed above, we have always used $\delta_{\min } \ll \delta_{0}$ in order to allow the direction of the initial perturbation to align with the most unstable phase-space direction, and hence to be able to recover the largest Lyapunov exponent in the limit of infinitesimal perturbations. In Fig. 2 (curve with filled triangles) we show what happens when this prescription is relaxed. In this case, the value of $\lambda(\delta)$ is underestimated for $\delta \sim \delta_{\min }$. This is due to the fact that the perturbation is not along the most unstable direction. The process of alignment of the perturbation along the unstable direction may result in a decrease of the distance between the two trajectories at initial times (i.e., at small $\delta$ ), leading to a lower value of $\lambda(\delta)$. The computation of $\lambda(\delta)$ without relaxation can indeed be more appropriate for characterizing short time predictability with large initial error, but we cannot expect, in this case, to asymptotically recover the largest Lyapunov exponent.

From these results, it is apparent that the effects of the fastest dynamics are seen only when the size of the perturbation is small enough. As a consequence, one has that the predictability for finite-size perturbations may be unaffected by the particular parametrization of the faster scales. To confirm this inference, in Fig. 3 we show $\lambda(\delta)$ for a case where in the evolution of the perturbed trajectory $\left(x_{\mathrm{s}}^{\prime}, x_{\mathrm{f}}^{\prime}\right)$ the fast variable $x_{\mathrm{f}}^{\prime}$ is replaced by a sequence of random numbers uniformly distributed in the interval $[0,1]$ (curve with filled triangles). The fact that $x_{\mathrm{f}}^{\prime}$ is now a random variable can be detected only for small enough $\delta$ (where $\lambda(\delta)$ is ruled by a logarithmic law, see [19]). The charactrization of the large-scale dynamics, however, is unaffected by the incorrect parametrization of the fast dynamics. 


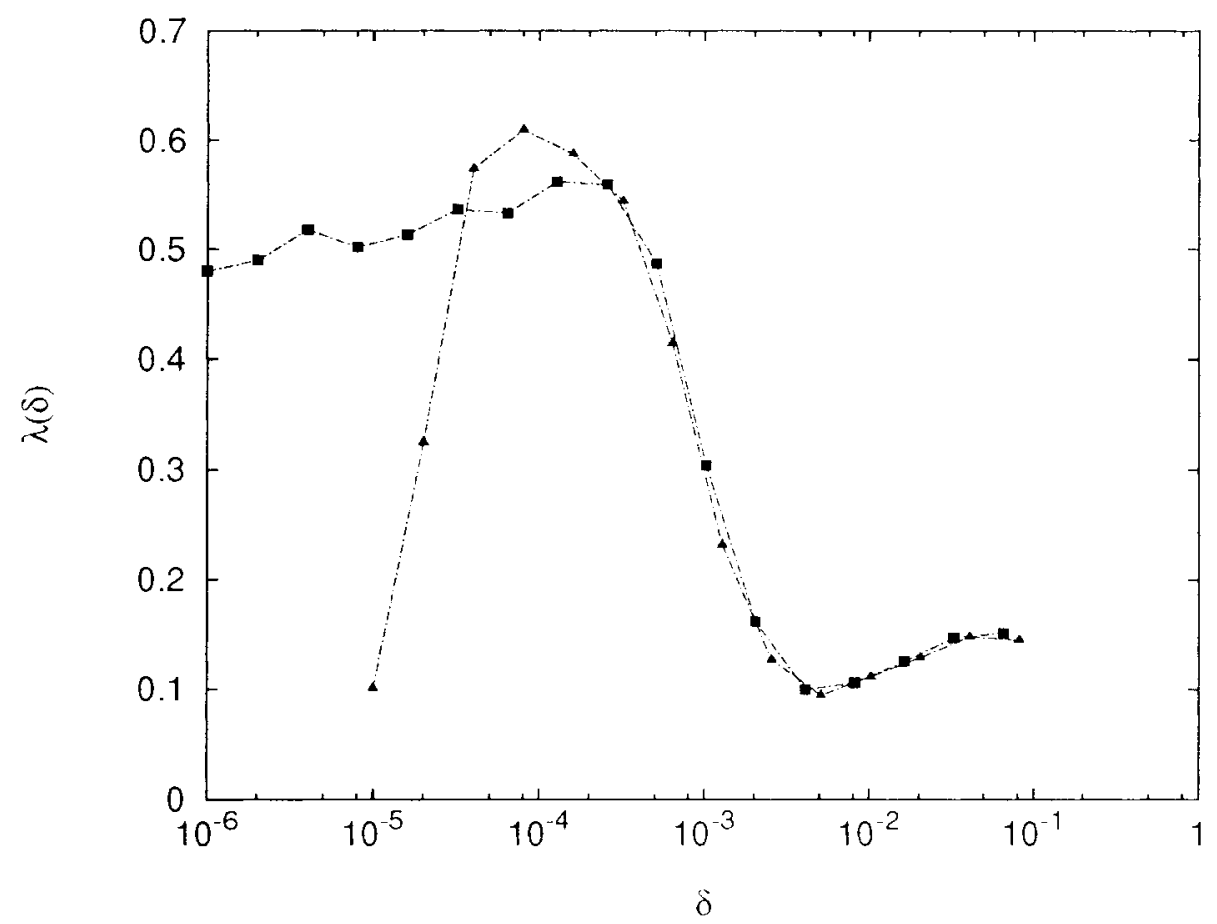

Fig. 2. The FSLE, $\lambda(\delta)$, as a function of $\delta$ for the coupled maps (3) and (4) with the same parameters as in Fig. 1. The filled squares refer to the results obtained with $\delta_{\min }=10^{-9}$ and $\delta_{0}=10^{-6}$ (same curve as in Fig. 1). The filled triangles show the behavior of $\lambda(\delta)$ when $\delta_{\min }=\delta_{0}=10^{-5}$ and the perturbation did not start along the most expanding direction.

Note, also, an interesting point. The results in Fig. 1 indicate that the small-scale dynamics is correctly recovered when a one-dimensional time series is used $\left(x_{\mathrm{s}}(n)\right)$, even though the full dynamics is two-dimensional $\left(x_{\mathrm{s}}(n), x_{\mathrm{f}}(n)\right)$. This event, possibly surprising at first sight, is due to the fact that the perturbed trajectory has been obtained by acting on the full phase space of the system; i.e., the perturbation has been made on both $x_{\mathrm{s}}(n)$ and $x_{\mathrm{f}}(n)$. When only $x_{\mathrm{s}}(n)$ is perturbed, as in the curve shown in Fig. 3, it is not possible to recover the fast small-scale dynamics without resorting to methods such as the time-embedding technique. In this case, however, other problems appear, as discussed in section.

\subsection{Coupled Lorenz models}

To illustrate the application of the FSLE technique to the case of continuous-time dynamical systems, here we consider a system obtained by coupling two Lorenz [24] models having time scales that differ by a factor $a$.

The slow subsystem is coupled through the Rayleigh number $R$ to the fast one; for simplicity, the fast subsystem does not feel any feedback from the slow one. More generic (small) couplings do not qualitatively change the results, see [20]. The equations for the whole system are

$$
\begin{aligned}
& \frac{\mathrm{d} x_{\mathrm{s}}}{\mathrm{d} t}=-\sigma x_{\mathrm{s}}+\sigma y_{\mathrm{s}}, \quad \frac{\mathrm{d} y_{\mathrm{s}}}{\mathrm{d} t}=-x_{\mathrm{s}} y_{\mathrm{s}}+\left(R+\epsilon z_{\mathrm{f}}\right) x_{\mathrm{s}}-y_{\mathrm{s}}, \quad \frac{\mathrm{d} z_{\mathrm{s}}}{\mathrm{d} t}=x_{\mathrm{s}} y_{\mathrm{s}}-b z_{\mathrm{s}}, \\
& \frac{\mathrm{d} x_{\mathrm{f}}}{\mathrm{d} t}=\left(-\sigma x_{\mathrm{f}}+\sigma y_{\mathrm{f}}\right) \cdot a, \quad \frac{\mathrm{d} y_{\mathrm{f}}}{\mathrm{d} t}=\left(-x_{\mathrm{f}} y_{\mathrm{f}}+R x_{\mathrm{f}}-y_{\mathrm{f}}\right) \cdot a, \quad \frac{\mathrm{d} z_{\mathrm{f}}}{\mathrm{d} t}=\left(x_{\mathrm{f}} y_{\mathrm{f}}-b z_{\mathrm{f}}\right) \cdot a,
\end{aligned}
$$

where the parameter $\epsilon$ controls the strength of the coupling and $a$ is the relative timescale. 


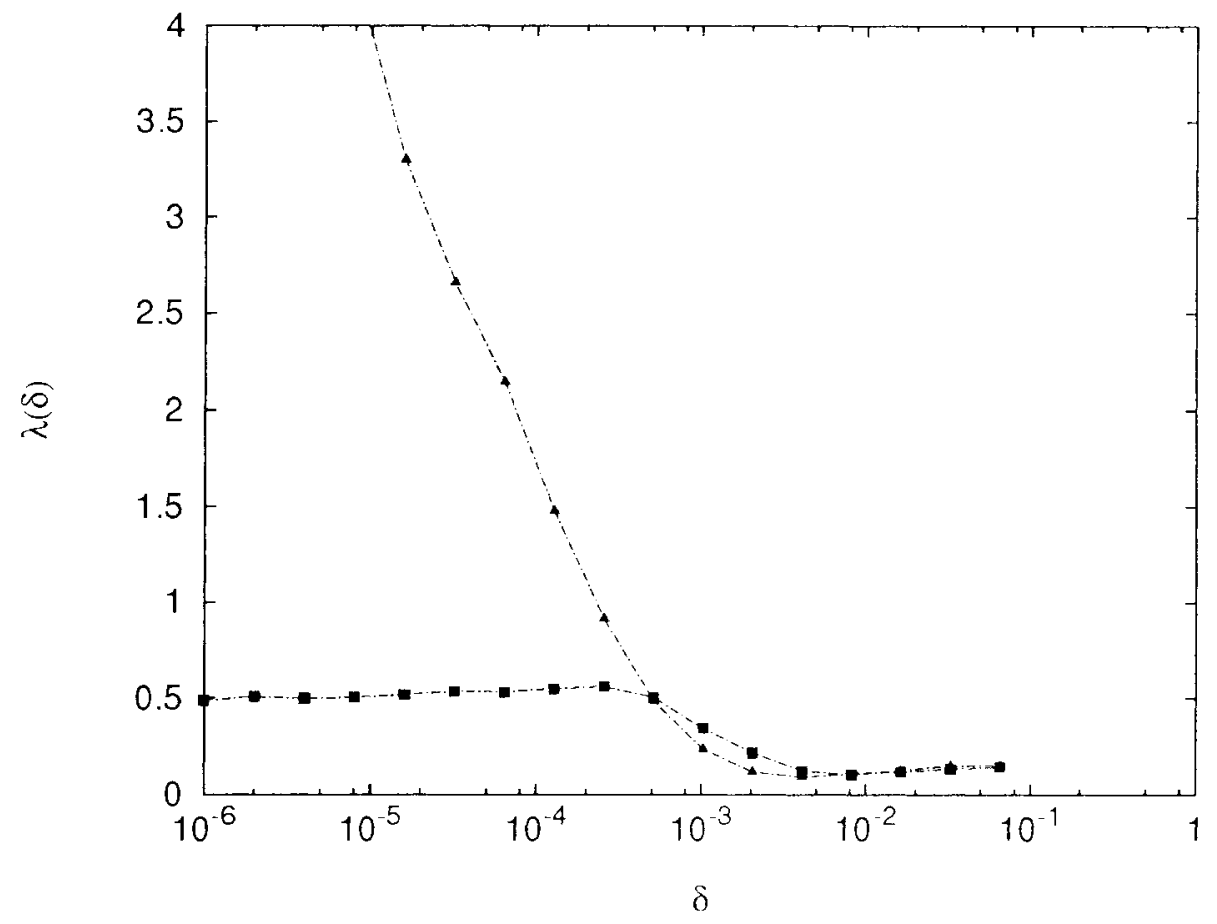

Fig. 3. The FSLE, $\lambda(\delta)$, as a function of $\delta$ for the coupled maps (3) and (4). The parameters are as in Fig. 1. The filled squares are as in Fig. 1 . The filled triangles refer to the case where the fast variables are replaced by a sequence of random numbers uniformly distributed in $[0,1]$.

In the first type of simulations, both the reference and the perturbed trajectories evolve with the same equations of motion (7). Again, we assume that only the slow variables are accessible; the norm is defined as the Euclidean distance in the three-dimensional space $\left(x_{\mathrm{s}}, y_{\mathrm{s}}, z_{\mathrm{s}}\right)$.

The parameters used in the numerical integrations are $R=45.92, \sigma=16, b=4$; the Lyapunov exponent of the slow subsystems is $\lambda_{\mathrm{s}} \simeq 1.5$. The ratio of the timescales of the two systems is $a=5$, hence $\lambda_{\mathrm{f}} \simeq \lambda_{\max } \simeq 7.5$. The results for $\epsilon=10^{-4}$ are shown in Fig. 4. As in the case of the coupled maps, $\lambda(\delta)$ displays two plateaus at $\lambda(\delta) \simeq \lambda_{\mathrm{f}}$ and $\lambda(\delta) \simeq \lambda_{\mathrm{s}}$, corresponding respectively to the fast and slow dynamics, and a transition region at $\delta \sim \epsilon$. Again, the small-scale dynamics (associated with the fast variables) can be recovered because we have perturbed the trajectory in the full, six-dimensional, phase space.

To investigate the role of small-scale parametrization, also for this system we have considered a situation where the "true" dynamics of the fast variables $x_{\mathrm{f}}^{\prime}, y_{\mathrm{f}}^{\prime}, z_{\mathrm{f}}^{\prime}$ in the perturbation is replaced by a stochastic process, i.e.

$$
\frac{\mathrm{d} x_{\mathrm{s}}^{\prime}}{\mathrm{d} t}=-\sigma x_{\mathrm{s}}^{\prime}+\sigma y_{\mathrm{s}}^{\prime}, \quad \frac{\mathrm{d} y_{\mathrm{s}}^{\prime}}{\mathrm{d} t}=-x_{\mathrm{s}}^{\prime} y_{\mathrm{s}}^{\prime}+(R+\epsilon \eta) x_{\mathrm{s}}^{\prime}-y_{\mathrm{s}}^{\prime}, \quad \frac{\mathrm{d} z_{\mathrm{s}}^{\prime}}{\mathrm{d} t}=x_{\mathrm{s}}^{\prime} y_{\mathrm{s}}^{\prime}-b z_{\mathrm{s}}^{\prime}
$$

where $\eta$ is a Gaussian white noise process with variance equal to that of the fast component in the original system (7). Analogously, we have considered a case where the fast dynamics of the perturbation is simply neglected; this corresponds to taking $\epsilon=0$ in the evolution equations for the perturbations $x_{\mathrm{s}}^{\prime}, y_{\mathrm{s}}^{\prime}, z_{\mathrm{s}}^{\prime}$. The two corresponding curves of $\lambda(\delta)$ are shown in Fig. 4 (filled triangles and open diamonds).

The results shown in Fig. 4 confirm that the estimate of $\lambda(\delta)$ for the slow variables is practically unaffected, for large values of $\delta$, by the details of the fast dynamics. A similar result was obtained in [25] for the chaotic or stochastic 


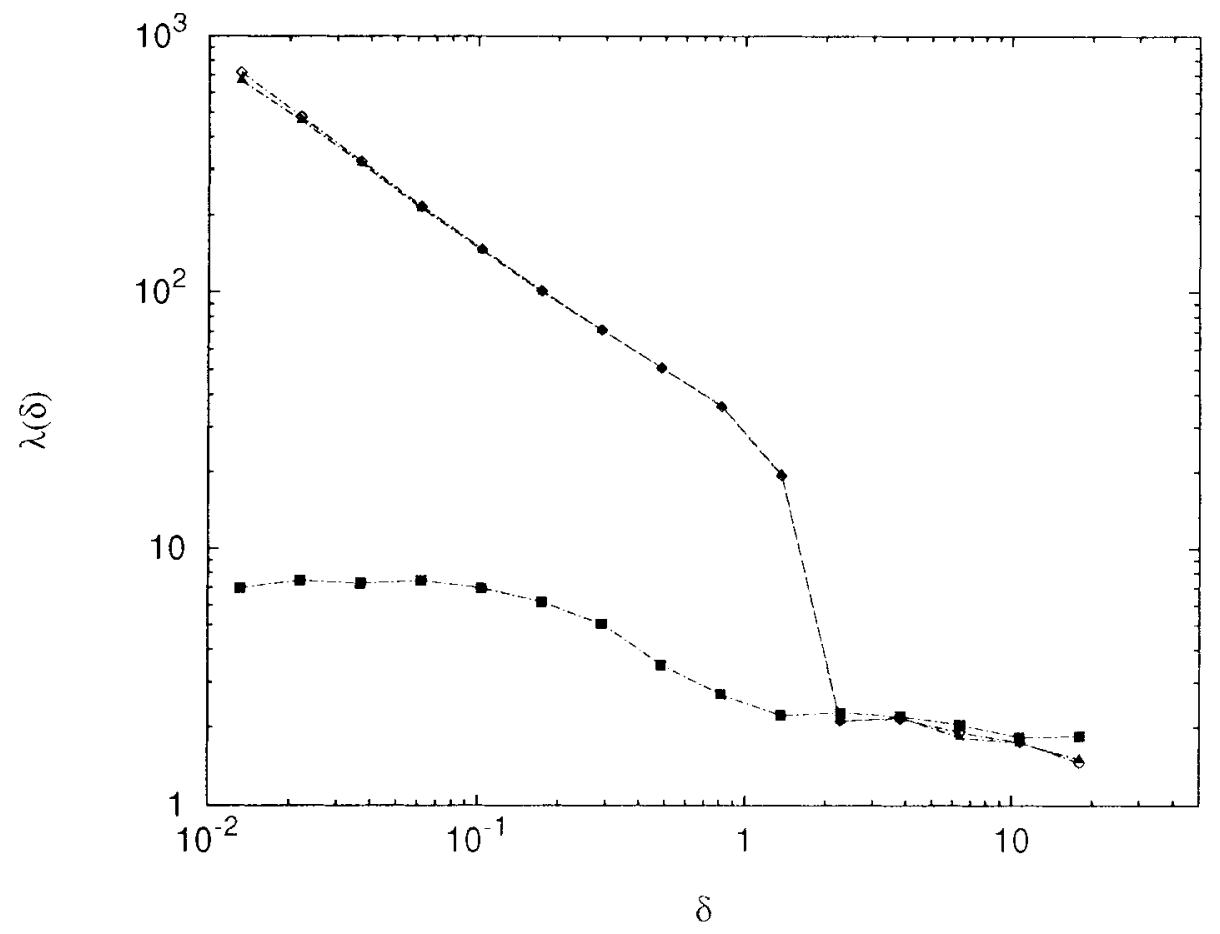

Fig. 4. The FSLE, $\lambda(\delta)$, as a function of $\delta$ for the coupled Lorenz systems (7) with $R=45.92, \sigma=16, b=4$ and coupling $\epsilon=10^{-4}$ (filled squares). The filled triangles indicate the results obtained when the perturbed trajectory evolves according to the modified dynamics (8) with $\epsilon=10^{-4}$. The open diamonds represent the case $\epsilon=0$ in the perturbed trajectory (no fast dynamics).

resonance of a driven nonlinear oscillator. In the present situation, one may even neglect the fast dynamics, and still obtain a reliable estimate of the slow evolution (and of the Lyapunov exponent associated with the slow variables). In particular, the lack of knowledge of the fast dynamics has an effect which is similar to that associated with the presence of noise. The inadequacy of the parametrization for scales smaller than $\epsilon$ is reflected, in both cases, in a large value of the FSLE, i.e., in a poor predictability of the phase-space dynamics on small scales. At larger scales and slower times, the FSLE coincides with the Lyapunov exponent of the (uncoupled) slow subsystem. This defines the predictive skill of the "incomplete model" on those scales.

\section{Computation of the FSLE from measured data}

In the case of measured time series, it is not usually possible to have access to the whole set of variables describing the system. Consistent with these limitations, here we suppose that only one time series of a scalar observable quantity $h_{n}$, function of the slow phase-space variables of the system, is given. Additionally, in most experimental situation, the time series of $h_{n}$ is characterized by limited statistics.

The first step is the procedure of phase-space reconstruction. The time-embedding method [6,7] allows to reconstruct a pseudo-phase-space with dimension $M$, by using time delay coordinates of the observed variable. A vector in this phase space is then defined as

$$
\mathbf{X}_{n}=\left(h_{n}, h_{n-\tau}, \ldots, h_{n-(M-1) \tau}\right),
$$




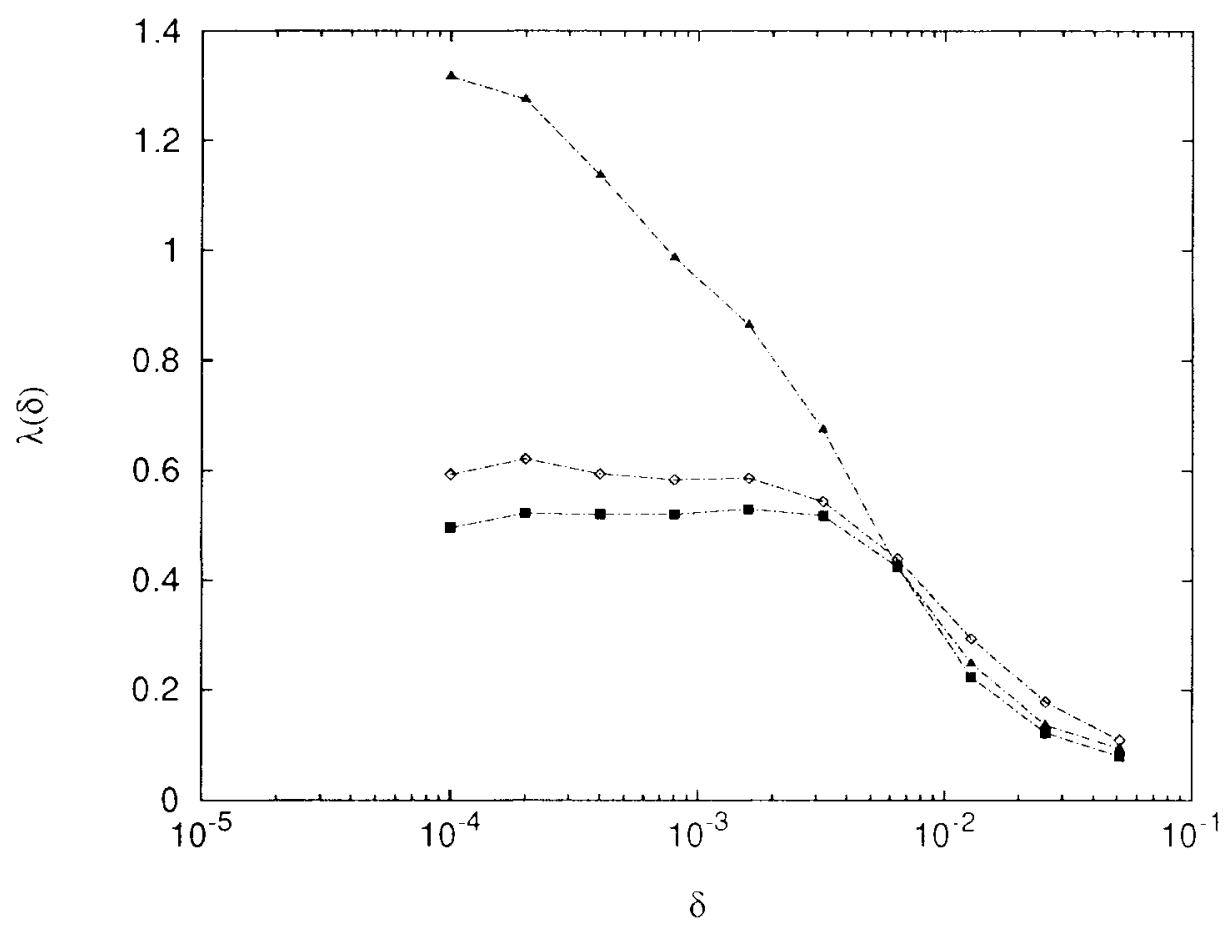

Fig. 5. The FSLE, $\lambda(\delta)$, as a function of $\delta$ for the time series $x(n)$ obtained from the coupled maps (3) and (4) with $\delta_{\min }=10^{-5}, \delta_{0}=10^{-4}$ and coupling $\epsilon=0.02$ (filled squares). The triangles indicate the results for an embedding dimension $M=1$ and the diamonds for $M=2$. The number of realizations used for each point in the FSLE is $10^{4}$. The total number of point in the time series is $10^{8}$ for the case $M=1$ and more than $10^{10}$ for $M=2$.

where $\tau$ is a suitably chosen time delay, see e.g. [2,3] for a discussion on the optimal choice of $\tau$. The method for computing the experimental FSLE is then a simple modification of the standard algorithm for the Lyapunov exponent [10] which measures the average separation between trajectories in the embedding space.

For each reconstructed vector $\mathbf{X}_{n}$, its nearest neighbor $\mathbf{X}_{m}$ is determined. If the separation $\delta=\left|\mathbf{X}_{n}-\mathbf{X}_{m}\right|$ is smaller that a given threshold $\delta_{\min }$, the trajectories starting from $\mathbf{X}_{n}$ and $\mathbf{X}_{m}$ are used to compute the FSLE, according to the algorithm discussed above. As in the case of maps, the trajectory is not continuous in time and one has to adopt the definition (5).

Also in this case we require $\delta_{\min }$ to be considerably smaller than $\delta_{0}$, to allow the vector separating the two trajectories to align with the maximally expanding direction. Clearly, this may severely limit the available statistics. A trivial geometrical argument shows that the probability of finding two points at a given distance in the embedding space becomes extremely small for high embedding dimensions. We have found this lack of statistics to be the most important limitation that prevents from taking the limit $\delta \rightarrow 0$, and thus from estimating the largest Lyapunov exponent. On the other hand, the statistics grows with the perturbation threshold $\delta_{j}$, we may thus expect to be able to compute $\lambda\left(\delta_{j}\right)$ for sufficiently large values of $\delta_{j}$. Another crucial point is related to the fact that the number of degrees of freedom which participate in the slow dynamics is (usually much) smaller than the total number of excited modes. Thus, the embedding dimension which is required for estimating $\lambda(\delta)$ for moderate values of $\delta$ is smaller than that needed for estimating the largest Lyapunov exponent.

As a first example, we consider the case where the signal $h_{n}$ is generated by the component $x_{\mathrm{s}}(n)$ of the coupled maps model (3). The coupling parameter is herein chosen to be $\epsilon=0.02$ (larger than that used in Section 3), in order to be able to study the small-scale behavior at $\delta<\epsilon$ even with moderate statistics. The results are shown in 


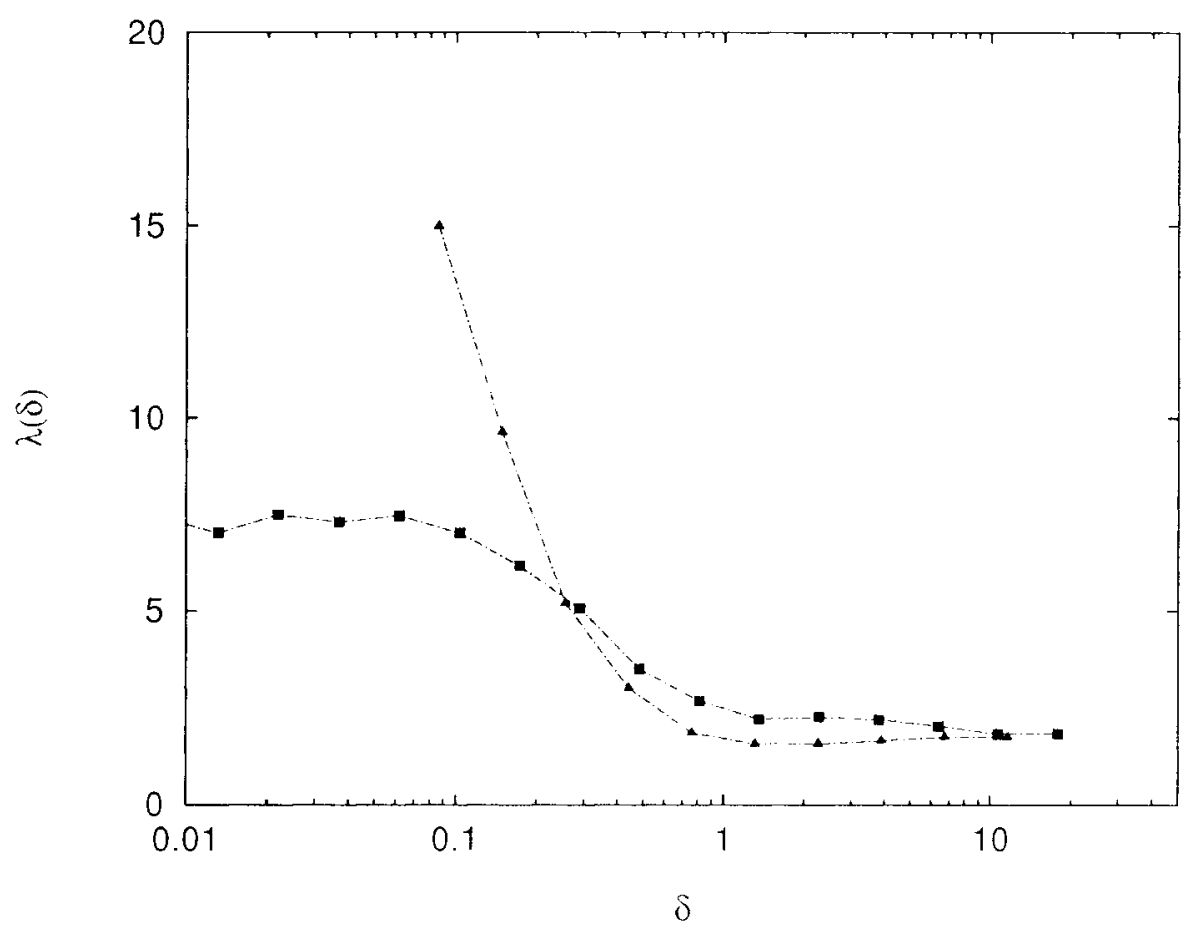

Fig. 6. The FSLE, $\lambda(\delta)$, as a function of $\delta$ for the time series $x_{\mathrm{s}}(t)$ obtained from the coupled Lorenz models (7) with coupling $\epsilon=0.05$ (filled squares). The filled triangles indicate the results for an embedding dimension $M=3$, time delay $\tau=0.02$ and a total number of points $N=500000$.

Fig. 5. The three curves show the FSLE as obtained in Section 3 (filled squares), and that from the time-embedding method with embedding dimensions $M=1$ (filled triangles) and $M=2$ (open diamonds) with time delay $\tau=1$. As expected [6,7], the computation of the Lyapunov exponent requires in this case an embedding dimension $M=2$ in order to resolve the fast dynamics. In fact, at variance with the results shown in Section 3, the one-dimensional time series is not enough here because we have no control on the perturbation in the fast variable. It is interesting, however, that one can obtain $\lambda(\delta)$ for large $\delta$ already with an embedding dimension which reflects the dimensionality of the slow system $(M=1)$.

Note that, in order to have good statistics, each point in Fig. 5 has been obtained by averaging (5) over $10^{4}$ samples. This requires a time series of about $10^{8}$ points for $M=1$ and more than $10^{10}$ points in the case $M=2$ to resolve the small scales at $\delta \sim 10^{-4}$. Thus, although it is in principle possible to extract information on the fast dynamics and on the largest Lyapunov exponent from a measured time series, the statistics required is so prohibitive that this procedure may be infeasible in realistic situations. On the other hand, it is possible to extract information on the large-scale Lyapunov exponent with an embedding dimension of the order of the number of degrees of freedom involved in the slow dynamics.

We observe that this result could not be obtained by simply neglecting the fast component as in a measured time series one has no direct access to the equations of motion.

As a second example, we now apply the same machinery to the case of the two coupled Lorenz systems described by Eq. (7). Fig. 6 shows the results obtained by using the variable $x_{\mathrm{s}}$ of (7), with $M=3, \tau=0.02$ and a total number of $N=500000$ points in the time series. The coupling constant between the models is now $\epsilon=0.05$. The perturbation threshold is fixed as $\delta_{\min }=0.005$ and $\delta_{0}=0.05$. The plateau corresponding to the large scales 
is clearly visible, while, in spite of the large number of points, the contribution of the fast system is not resolved. Clearly, this would require a larger embedding dimension and an increase of the smallest resolved value of $\delta$, at the cost of an unrealistic increase of the number of points in the time series.

\section{Conclusions}

In this work we have discussed how to use the method of the finite size Lyapunov exponent (FSLE) for determining the slow dynamics of systems with many different characteristic times. In particular, we have considered the case when full access to the slow dynamics is allowed, and the more realistic case when just one scalar time series of a slow variable has been measured. The basic idea is to compute the FSLE, $\lambda(\delta)$, as a function of $\delta$ in the framework of the embedding technique. In this case, the behavior of $\lambda(\delta)$ at large values of $\delta$ gives information on the Lyapunov exponents associated with the slow dynamics.

By contrast, the behavior of $\lambda(\delta)$ for small value of $\delta$ gives information on the fast dynamics. By considering the limit of $\lambda(\delta)$ for $\delta \rightarrow 0$, it is possible, at least in principle, to discriminate between "stochastic" systems and "chaotic but deterministic" ones. For an accurate estimate of $\lambda(\delta)$ at small $\delta$ 's, however, it is necessary to use a very large number of points in the time series. This fact makes practically infeasible, the calculation of the largest Lyapunov exponent (associated with the fast dynamics) in most complex dynamical systems.

Nevertheless, very often the slow, large-scale dynamics is the most interesting one physically. The results obtained here indicate that the slow dynamics may be satisfactorily detected even with a limited number of points and a moderate embedding dimension. In these systems, one can thus obtain a satisfactory prediction for the slow, physically interesting scales even when access to the (much more unpredictable) fast scales is not available. This also indicates that, at least in the examples considered here, the parametrization of the fast timescales seems not to be crucial, as the internal dynamics of the slow modes plays the dominant role.

One could wonder, then, how general the results presented in this paper are. Previous works on more complex theoretical models [20] indicate that the crucial point is not the dimensionality of the system or the details of the couplings, but rather the existence of well separated, weakly interacting, scales. In the present work we have shown that the FSLE technique may be successfully applied also in the case of measured time series.

\section{Acknowledgements}

This work has been partially supported by the CNR research project "Climate Variability and Predictability" and by the INFN "Iniziativa Specifica Meccanica Statistica" FI11. GB thanks the Istituto di Cosmogeofisica del CNR for their hospitality.

\section{References}

[1] P.G. Drazin, G.P. King (Eds.), Interpretation of Time Series from Nonlinear Systems Physica D 58 (1992).

[2] E. Ott, T. Sauer, J.A. Yorke, Coping with Chaos, Wiley, New York, 1994.

[3] H.D.I. Abarbanel, R. Brown, J.J. Sidorowich, L.Sh. Tsimring, Rev. Modern Phys. 65 (1993) 1331.

[4] H. Kantz, in: J. Parisi, S.C. Müller, W. Zimmermann (Eds.), Nonlinear Physics of Complex Systems, Springer, Berlin, 1996. p. 213.

[5] J.-P. Eckmann, D. Ruelle, Rev. Modern Phys. 57 (1985) 617.

[6] N. Packard, J. Crutchfield, J.D. Farmer, R. Shaw, Phys. Rev. Lett. 45 (1981) 712.

[7] F. Takens, in: D. Rand, L.S. Young (Eds.), Dynamical Systems and Turbulence, Warwick 1980, Springer, Berlin, 1981 , p. 366.

[8] P. Grassberger, I. Procaccia, Phys. Rev. Lett. 50 (1983) 346.

[9] P. Grassberger, I. Procaccia, Phys. Rev. A 28 (1983) 2591. 
[10] A. Wolf, J.B. Swift, H. Swinney, J.A. Vastano, Physica D 16 (1985) 285.

[11] L.A. Smith, Phys. Lett. A 133 (1988) 283.

[12] J.-P. Eckmann, D. Ruelle, Physica D 56 (1992) 185.

[13] A.R. Osborne, A. Provenzale, Physica D 35 (1989) 357.

[14] R. Vio, S. Cristiani, O. Lessi, A. Provenzale, Astrophys. J. 391 (1992) 518.

[15] A. Provenzale, L.A. Smith, R. Vio, G. Murante, Physica D 58 (1992) 31.

[16] N. Platt, E.A. Spiegel, C. Tresser, Phys. Rev. Lett. 70 (1994) 279.

[17] J. Graf von Hardenberg, F. Paparella, N. Platt, A. Provenzale, E.A. Spiegel, C. Tresser, Phys. Rev. E 50 (1997) 58.

[18] G. Boffetta, G. Paladin, A. Vulpiani, J. Phys. A 29 (1996) 2291.

[19] E. Aurell, G. Boffetta, A. Crisanti, G. Paladin, A. Vulpiani, Phys. Rev. Lett. 77 (1996) 1262; J. Phys. A 30 (1997) 1.

[20] G. Boffetta, P. Giuliani, G. Paladin, A. Vulpiani, J. Atmos. Sci. (1997) in press.

[21] E.N. Lorenz, Predictability - a problem partly solved, Proceeding of the Seminar on predictability, Reading, UK, European Centre for Medium-Range Weather Forecast, 1996, p. 1.

[22] A.N. Kolmogorov, IRE Trans. Inf. Theory 1 (1956) 102.

[23] P. Gaspard, X.J. Wang, Phys. Rep. 235 (1993) 291.

[24] E. Lorenz, J. Atmos. Sci. 20 (1963) 130.

[25] J. Graf von Hardenberg, F. Paparella, A. Provenzale, E.A. Spiegel, in: J.R. Buchler, H. Kandrup (Eds.), Nonlinear Signal and Image Analysis, Annals NY Acad. Sci., 1997, p. 79. 